PROCEEDINGS OF THE

AMERICAN MATHEMATICAL SOCIETY

Volume 132, Number 5, Pages 1517-1524

S 0002-9939(03)07258-7

Article electronically published on October 3, 2003

\title{
ADJOINT RESTRICTION ESTIMATES AND SCALING ON SPHERES
}

\author{
BASSAM SHAYYA
}

(Communicated by Andreas Seeger)

\begin{abstract}
We test the restriction conjecture, in its adjoint form, against a class of measures $\phi_{\delta} d \sigma$ on the sphere $\mathbf{S}^{n-1}$. The densities $\phi_{\delta}$ are smoothed out characteristic functions of $\delta^{a_{2}} \times \delta^{a_{3}} \times \cdots \times \delta^{a_{n}}$ rectangular caps on $\mathbf{S}^{n-1}$, where $a_{2}, a_{3}, \ldots, a_{n}$ are fixed nonnegative numbers.
\end{abstract}

\section{INTRODUCTION}

Let $n \geq 2,1 \leq p \leq \infty, 2 n /(n-1)<q<\infty$, and consider the adjoint restriction estimate

$$
\|\widehat{f d \sigma}\|_{L^{q}\left(\mathbb{R}^{n}\right)} \lesssim\|f\|_{L^{p}\left(\mathbb{S}^{n-1}\right)}
$$

for $f \in L^{p}\left(\mathbb{S}^{n-1}\right)$, where $\sigma$ is the surface measure on the unit sphere $\mathbb{S}^{n-1} \subset \mathbb{R}^{n}$. The restriction conjecture asserts that (1) holds whenever

$$
\frac{n-1}{p}+\frac{n+1}{q} \leq n-1 \text {. }
$$

Fix nonnegative numbers $a_{1} \leq a_{2} \leq \cdots \leq a_{n}$ and a $C^{\infty}$ function $\phi$ supported in a ball in $\mathbb{R}^{n}$ of center 0 and small 1$]$ radius $c_{0}$. For $\theta \in \mathbb{S}^{n-1}$ and $0<\delta \leq 1$, define

$$
\phi_{\delta}(\theta)=\phi\left(\left(\theta_{1}-1\right) / \delta^{a_{1}}, \theta_{2} / \delta^{a_{2}}, \ldots, \theta_{n} / \delta^{a_{n}}\right) .
$$

Then $\phi_{\delta}$ is supported in a rectangular cap on $\mathbb{S}^{n-1}$ of dimensions $c_{0} \delta^{a_{2}} \times c_{0} \delta^{a_{3}} \times \cdots \times$ $c_{0} \delta^{a_{n}}$ and center $e_{1}=(1,0, \ldots, 0),\left\|\phi_{\delta}\right\|_{L^{p}\left(\mathbb{S}^{n-1}\right)} \lesssim \delta^{a / p}$ where $a=a_{2}+a_{3}+\cdots+a_{n}$, and (1) implies

$$
\left\|\widehat{\phi_{\delta} d \sigma}\right\|_{L^{q}\left(\mathbb{R}^{n}\right)} \lesssim \delta^{a / p}
$$

with the implicit constant 2 independent of $\delta$.

The purpose of this paper is to find necessary and sufficient conditions on $p$ and $q$ for which the estimate (3) holds.

In the case $a_{1}=\cdots=a_{n}=1$, a well-known argument, due to A. Knapp, shows that (2) is a necessary condition for (11) to hold by showing that (2) is necessary for the weaker estimate (3) when $\phi$ is nonnegative and satisfies $\phi(x) \gtrsim 1$ for $|x| \lesssim 1$. The argument goes as follows. $\left|\widehat{\phi_{\delta} d \sigma}\right| \approx \delta^{n-1}$ on a $C \delta^{-2} \times C \delta^{-1} \times \cdots \times C \delta^{-1}$ box

Received by the editors May 20, 2002 and, in revised form, January 28, 2003.

2000 Mathematics Subject Classification. Primary 42B10, 42B15.

${ }^{1}$ It suffices for our purposes to take $c_{0} \leq 1 /(100 n)^{2}$.

${ }^{2}$ All constants in this paper are positive and independent of $\delta$. 
(for an appropriate constant $C$ ) centered at the origin in $\mathbb{R}^{n}$ with the long side parallel to $e_{1}$. Inserting this into (3) one obtains

$$
\frac{\delta^{n-1}}{\delta^{(n+1) / q}} \lesssim \delta^{\frac{n-1}{p}}
$$

and (22) follows by letting $\delta \rightarrow 0$. For further details we refer to [1].

Repeating Knapp's argument in the general case, we see that $\left|\widehat{\phi_{\delta} d \sigma}\right| \approx \delta^{a}$ on a $C \delta^{-2 a_{2}} \times C \delta^{-a_{2}} \times \cdots \times C \delta^{-a_{n}}$ box centered at the origin with the $\delta^{-2 a_{2}}$ side parallel to $e_{1}$; so for (3) to hold, $p$ and $q$ must satisfy

$$
\frac{a}{p}+\frac{a+2 a_{2}}{q} \leq a \text {. }
$$

Our first result is the following improvement on (4).

Theorem 1. Assume $\phi$ is nonnegative and $\phi(x) \gtrsim 1$ for $|x| \lesssim 1$. Suppose $1 \leq p \leq$ $\infty$ and $1 \leq q<\infty$. If (33) holds, then $q>2 n /(n-1),(p, q)$ satisfies (4), and

$$
\frac{a}{p}+\frac{2 n a_{n}-a}{q} \leq(n-1) a_{n}
$$

We then turn matters around and try to find a range of $(p, q)$ for which (3) holds. Of course, one would like to prove that (3) holds for the full range of $(p, q)$ in Theorem 1 which is larger than that of (2), but we shall be able to do this (up to the sharp line) only in the case $a_{2}=\cdots=a_{n}$ (when Theorem 11 and (2) agree). In the general case we obtain a range of $(p, q)$ smaller than that in (2), but, at any rate, larger than what is already known for the restriction problem.

Theorem 2. Suppose that $1 \leq p \leq \infty$ and $2 n /(n-1)<q<\infty$. Then (3) holds whenever

$$
\frac{n-1}{p}+\frac{n+1}{q} \leq n-1 \quad \text { and } \quad \frac{a}{p}+\frac{(n+1) a_{n}}{q}<\frac{a+(n-1) a_{n}}{2} .
$$

In particular, if $a_{2}=\cdots=a_{n}$, then (3) holds whenever

$$
\frac{n-1}{p}+\frac{n+1}{q}<n-1 \text {. }
$$

The proofs of Theorems 1 and 2 depend heavily on the smoothness of the function $\phi$ that will be used to obtain an asymptotic expansion formula for $\widehat{\phi_{\delta} d \sigma}$. This will be done in Section 2. In Section 3 we prove Theorem 1 and in Section 4, Theorem 2

\section{ACKNOWLEDGMENT}

The author wishes to thank the referee for many helpful suggestions that led, in particular, to strengthening Proposition 1 to its present form.

\section{Main estimate}

In this section we use the method of stationary phase to produce an asymptotic expansion formula for $\widehat{\phi_{\delta} d \sigma}$ with the dependence of the various terms on $\delta$ explicitly described.

Recall that $\phi$ is supported in a ball in $\mathbb{R}^{n}$ of center 0 and small radius $c_{0}$. 
Proposition 1. Let $c=2 c_{0} / \sqrt{1-c_{0}^{2}}$ and

$$
\Omega_{\delta}=\left\{\theta \in \mathbb{S}^{n-1}:\left|\theta_{j}\right| \leq c \delta^{a_{j}} \text { for } j=2, \ldots, n\right\} .
$$

Then:

(i) For $\xi=|\xi| \xi^{\prime}$ with $|\xi|>0$ and $\xi^{\prime} \in \Omega_{\delta}$, we have

$$
\widehat{\phi_{\delta} d \sigma}(\xi)=\frac{1}{|\xi|^{\frac{n-1}{2}}}\left(\phi_{\delta}\left(\xi^{\prime}\right) e^{-2 \pi i\left(|\xi|-\frac{n-1}{8}\right)}+\phi_{\delta}\left(-\xi^{\prime}\right) e^{2 \pi i\left(|\xi|-\frac{n-1}{8}\right)}+O\left(\frac{1}{\delta^{2 a_{n}}|\xi|}\right)\right) \text {. }
$$

(ii) For $\xi=|\xi| \xi^{\prime}$ with $|\xi|>0$ and $\xi^{\prime} \notin \Omega_{\delta}$, we have

$$
\left|\widehat{\phi_{\delta} d \sigma}(\xi)\right| \lesssim \frac{\delta^{a}}{\left(\delta^{2 a_{n}}|\xi|\right)^{N}}
$$

for any positive integer $N$.

Proof. A point $x \in \mathbb{R}^{n-1}$ will be written as $x=\left(x_{2}, \ldots, x_{n}\right)$.

Let $B=B(0,1 / 2)=\left\{x \in \mathbb{R}^{n-1}:|x|<1 / 2\right\}$ and consider the local coordinate $F: B \rightarrow \mathbb{S}^{n-1}$ given by

$$
F(x)=\left(\sqrt{1-|x|^{2}}, x\right) .
$$

Then

$$
\widehat{\phi_{\delta} d \sigma}(\xi)=\int_{\mathbb{S}^{n-1}} e^{-2 \pi i \xi \cdot \theta} \phi_{\delta}(\theta) d \sigma(\theta)=\int_{B} e^{-2 \pi i \xi \cdot F(x)} \phi_{\delta}(F(x)) q(x) d x,
$$

where $q$ is a real-valued $C^{\infty}$ function supported in $B$ with $q(0)=1$. Since the phase function in the above integral has no critical points in Supp $\phi_{\delta} \circ F$ when $\xi^{\prime} \notin\left(\operatorname{Supp} \phi_{\delta}\right) \cup\left(-\operatorname{Supp} \phi_{\delta}\right)$, part (ii) of the proposition follows from an easy argument of integration by parts. So we only need to consider the situation when $\xi^{\prime} \in \Omega_{\delta}$, i.e., when

$$
\left|\xi_{j}^{\prime}\right| \leq c \delta^{a_{j}} \quad \text { for } \quad j=2, \ldots, n .
$$

Clearly, we can also assume that $\xi_{1}^{\prime}>0$.

Set

$$
C_{\delta}=\left\{z \in \mathbb{R}^{n}: \frac{1}{2}<z_{1}<2 \text { and }\left|z_{j}\right| \leq c \delta^{a_{j}} \text { for } j=2, \ldots, n\right\},
$$

$\lambda=|\xi|$, and let $T$ be a rotation on $\mathbb{R}^{n}$ such that $T \xi^{\prime}=e_{1}$. Then $\xi^{\prime} \in C_{\delta} \cap \mathbb{S}^{n-1}$ and

$$
\widehat{\phi_{\delta} d \sigma}(\xi)=\int_{\mathbb{S}^{n-1}} e^{-2 \pi i \lambda e_{1} \cdot \theta} \psi(\theta) d \sigma(\theta),
$$

where $\psi(\theta)=\phi_{\delta}\left(T^{-1} \theta\right)$. Now consider the dilation group $\left\{A_{t}\right\}_{t>0}$ on $\mathbb{R}^{n}$ given by

$$
A_{t} z=\left(t^{a_{1}} z_{1}, \ldots, t^{a_{n}} z_{n}\right)
$$

and notice that

where

$$
\psi(\theta)=\phi_{\delta}^{\prime}(\theta)=\phi^{\prime}\left(A_{1 / \delta}\left(\theta-e_{1}\right)\right)
$$

$$
\phi^{\prime}(\theta)=\phi\left(A_{1 / \delta} T^{-1} A_{\delta} \theta+A_{1 / \delta}\left(\xi^{\prime}-e_{1}\right)\right) .
$$

To estimate the integral in (8), we shall require of the rotation $T$, in addition to $T \xi^{\prime}=e_{1}$, the following two properties:

- there is a constant $c^{\prime}$ such that

$$
T\left(C_{\delta}\right) \subset\left\{z \in \mathbb{R}^{n}: 0<z_{1}<3 \text { and }\left|z_{j}\right| \leq c^{\prime} \delta^{a_{j}} \text { for } j=2, \ldots, n\right\} ;
$$

- $\left\|A_{1 / \delta} T^{-1} A_{\delta}\right\| \lesssim 1$. 
The first property tells us that Supp $\phi^{\prime} \subset\left\{z \in \mathbb{R}^{n}:\left|z_{j}\right| \leq c^{\prime}\right.$ for $\left.j=2, \ldots, n\right\}$. The second guarantees that the partial derivatives of $\phi^{\prime}$ are bounded uniformly in $\delta$. To find such a rotation, put $\beta_{-1}=1$ and

$$
\beta_{j}=\sqrt{1-\left(\xi_{n-j}^{\prime}\right)^{2}-\left(\xi_{n-j+1}^{\prime}\right)^{2}-\cdots-\left(\xi_{n}^{\prime}\right)^{2}} \quad(j=0, \ldots, n-2),
$$

define rotations $S_{j}$ on $\mathbb{R}^{n}$ by

$$
S_{j}\left(e_{k}\right)= \begin{cases}\left(\beta_{j} / \beta_{j-1}\right) e_{1}+\left(\xi_{n-j}^{\prime} / \beta_{j-1}\right) e_{n-j} & \text { if } k=1, \\ -\left(\xi_{n-j}^{\prime} / \beta_{j-1}\right) e_{1}+\left(\beta_{j} / \beta_{j-1}\right) e_{n-j} & \text { if } k=n-j, \\ e_{k} & \text { if } k \neq 1, n-j\end{cases}
$$

(of course, $\left\{e_{1}, \ldots, e_{n}\right\}$ is the standard basis in $\mathbb{R}^{n}$ ), and put

$$
T^{-1}=S_{n-2} S_{n-3} \ldots S_{1} S_{0} .
$$

Then

$$
T^{-1} e_{k}=S_{n-2} \ldots S_{n-k}\left(e_{k}\right) \quad(k=2, \ldots, n),
$$

and since $S_{n-k}, \ldots, S_{n-2}$ do not change the last $n-k$ coordinates of any point in $\mathbb{R}^{n}$, it follows that the last $n-k$ coordinates of $T^{-1} e_{k}$ are all zeroes. On the other hand, since $\beta_{j} \geq \sqrt{1-(n-1) c^{2}}$ for $j=0, \ldots, n-2$, each of the first $k-1$ coordinates of $T^{-1} e_{k}$ is $\leq\left|\xi_{k}^{\prime}\right| /\left(1-(n-1) c^{2}\right) \leq c \delta^{a_{k}} /\left(1-(n-1) c^{2}\right)$. It is now an easy matter to verify that $T$ satisfies the required properties.

Going back to (8), we now have

$$
\widehat{\phi_{\delta} d \sigma}(\xi)=\int_{B} e^{-2 \pi i \lambda \sqrt{1-|x|^{2}}} \psi(F(x)) q(x) d x .
$$

Making the change of variables $x=G(u)=\left(G_{2}(u), \ldots, G_{n}(u)\right)$ with

$$
G_{j}(u)=u_{j} \sqrt{1-\frac{u_{j}^{2}}{4}-\frac{1}{2}\left(u_{j+1}^{2}+\cdots+u_{n}^{2}\right)} \quad(j=2, \ldots, n),
$$

we get

$$
\widehat{\phi_{\delta} d \sigma}(\xi)=\int_{U} e^{-2 \pi i \lambda \sqrt{1-|G(u)|^{2}}} \Phi(u) d u=e^{-2 \pi i \lambda} \int_{U} e^{\pi i \lambda|u|^{2}} \Phi(u) d u,
$$

where $U=G^{-1} B$ and $\Phi=(\psi \circ F \circ G)(q \circ G)\left|J_{G}\right|$. The observations we made before about Supp $\phi^{\prime}$ and its derivatives tell us that

$$
\text { Supp } \Phi \subset\left\{u \in \mathbb{R}^{n-1}:\left|u_{j}\right| \leq 2 c^{\prime} \delta^{a_{j}} \text { for } j=2, \ldots, n\right\}
$$

and

$$
\begin{aligned}
\left|D^{m} \Phi(u)\right| & \lesssim \sum_{m^{\prime} \leq m}\left|D^{m^{\prime}}(\psi \circ F \circ G)(u)\right| \\
& \lesssim \sum_{m^{\prime} \leq m} \frac{1}{\delta^{m^{\prime} \cdot\left(a_{2}, \ldots, a_{n}\right)}} \\
& \lesssim \frac{1}{\delta^{m \cdot\left(a_{2}, \ldots, a_{n}\right)}}
\end{aligned}
$$

for all $u \in U$ and every multi-index $m=\left(m_{2}, \ldots, m_{n}\right)$. Also,

$$
\Phi(0)=\psi\left(e_{1}\right) q(0)\left|J_{G}(0)\right|=\phi_{\delta}\left(\xi^{\prime}\right) .
$$

Since the distributional Fourier transform of $e^{\pi i \lambda|u|^{2}}$ is

$$
e^{\pi i \frac{n-1}{4}} \lambda^{-\frac{n-1}{2}} e^{-\pi i \lambda^{-1}|y|^{2}},
$$


it follows that

$$
\begin{aligned}
\widehat{\phi_{\delta} d \sigma}(\xi) & =e^{-2 \pi i\left(\lambda-\frac{n-1}{8}\right)} \lambda^{-\frac{n-1}{2}} \int e^{-\pi i \lambda^{-1}|y|^{2}} \widehat{\Phi}(y) d y \\
& =e^{-2 \pi i\left(\lambda-\frac{n-1}{8}\right)} \lambda^{-\frac{n-1}{2}} \Phi(0)+\lambda^{-\frac{n-1}{2}-1} E(\lambda)
\end{aligned}
$$

with

$$
E(\lambda)=e^{-2 \pi i\left(\lambda-\frac{n-1}{8}\right)} \lambda \int\left(e^{-\pi i \lambda^{-1}|y|^{2}}-1\right) \widehat{\Phi}(y) d y .
$$

To estimate this last integral, set $\Phi^{\delta}(u)=\Phi\left(A_{\delta} u\right)$, let $N>n$ be an integer, and observe that

$$
\begin{aligned}
& |E(\lambda)| \leq \int|y|^{2}|\widehat{\Phi}(y)| d y \\
& =\int|y|^{2}\left|\widehat{\Phi^{\delta}}\left(A_{\delta} y\right)\right| \delta^{a} d y \\
& =\int\left|A_{1 / \delta} v\right|^{2}\left|\widehat{\Phi^{\delta}}(v)\right| d v \\
& \leq \delta^{-2 a_{n}} \int|v|^{2}\left|\widehat{\Phi^{\delta}}(v)\right| d v \\
& \leq \quad \delta^{-2 a_{n}} \int \frac{(1+|v|)^{N+2}}{(1+|v|)^{N}}\left|\widehat{\Phi^{\delta}}(v)\right| d v \\
& \lesssim \delta^{-2 a_{n}} \sum_{|m| \leq N+2} \int \frac{1}{(1+|v|)^{N}}\left|v^{m} \widehat{\Phi^{\delta}}(v)\right| d v \\
& \approx \delta^{-2 a_{n}} \sum_{|m| \leq N+2} \int \frac{1}{(1+|v|)^{N}}\left|\widehat{D^{m} \Phi^{\delta}}(v)\right| d v \\
& \lesssim \delta^{-2 a_{n}} \sum_{|m| \leq N+2}\left\|\widehat{D^{m} \Phi^{\delta}}\right\|_{L^{\infty}\left(\mathbb{R}^{n-1}\right)} \\
& \leq \delta^{-2 a_{n}} \sum_{|m| \leq N+2}\left\|D^{m} \Phi^{\delta}\right\|_{L^{1}\left(\mathbb{R}^{n-1}\right)} \\
& \lesssim \delta^{-2 a_{n}} \sum_{|m| \leq N+2}\left\|D^{m} \Phi^{\delta}\right\|_{L^{\infty}\left(\mathbb{R}^{n-1}\right)} \\
& \lesssim \delta^{-2 a_{n}}
\end{aligned}
$$

where in the last two lines we have used (91) and (10).

\section{Proof of Theorem 1}

Choose a constant $c^{\prime}$ such that $\phi_{\delta} \gtrsim 1$ on the spherical cap

$$
C_{\delta}=\left\{\theta \in \mathbb{S}^{n-1}: \theta_{1}>0,\left|\theta_{2}\right| \leq c^{\prime} \delta^{a_{2}}, \ldots,\left|\theta_{n}\right| \leq c^{\prime} \delta^{a_{n}}\right\} .
$$

By Proposition 1, there is a constant $c^{\prime \prime}$ such that

$$
\left|\widehat{\phi_{\delta} d \sigma}(\xi)\right| \gtrsim \frac{1}{|\xi|^{\frac{n-1}{2}}}
$$


for $\xi=|\xi| \xi^{\prime}, \xi^{\prime} \in C_{\delta},|\xi| \geq c^{\prime \prime} \delta^{-2 a_{n}}$. Thus

$$
\begin{aligned}
\left\|\widehat{\phi_{\delta} d \sigma}\right\|_{L^{q}\left(\mathbb{R}^{n}\right)}^{q} & \geq \int_{c^{\prime \prime} \delta-2 a_{n}}^{\infty} \int_{C_{\delta}}\left|\widehat{\phi_{\delta} d \sigma}(r \theta)\right|^{q} r^{n-1} d \sigma(\theta) d r \\
& \gtrsim \sigma\left(C_{\delta}\right) \int_{c^{\prime \prime} \delta^{-2 a_{n}}}^{\infty} r^{n-1-(n-1)(q / 2)} d r .
\end{aligned}
$$

If $q \leq 2 n /(n-1)$, the above integral equals $\infty$, so that $\left\|\widehat{\phi_{\delta} d \sigma}\right\|_{L^{q}\left(\mathbb{R}^{n}\right)}=\infty$. If $q>2 n /(n-1)$, we get

$$
\left\|\widehat{\phi_{\delta} d \sigma}\right\|_{L^{q}\left(\mathbb{R}^{n}\right)} \gtrsim \delta^{a / q} \delta^{(n-1) a_{n}-2 n a_{n} / q} .
$$

Inserting this estimate into (3) we obtain

$$
\frac{a}{p}+\frac{2 n a_{n}-a}{q} \leq(n-1) a_{n}
$$

\section{Proof of Theorem 2}

Let $\epsilon>0$ and put $R=\delta^{-2 a_{n}-\epsilon}$. Also, let $B=B(0, R)$ be the ball in $\mathbb{R}^{n}$ of center 0 and radius $R$. Proposition 1 tells us that

$$
\begin{aligned}
\left\|\widehat{\phi_{\delta} d \sigma}\right\|_{L^{q}\left(B^{c}\right)} & \\
& \lesssim\left(\sigma\left(\Omega_{\delta}\right) \int_{R}^{\infty} \frac{r^{n-1}}{r^{\frac{n-1}{2} q}} d r\right)^{1 / q}+\delta^{-2 N a_{n}}\left(\sigma\left(\mathbb{S}^{n-1}-\Omega_{\delta}\right) \int_{R}^{\infty} \frac{r^{n-1}}{r^{N q}} d r\right)^{1 / q} \\
& \lesssim\left(\delta^{a} \int_{\delta^{-2 a_{n}}}^{\infty} r^{n-\frac{n-1}{2} q-1} d r\right)^{1 / q}+\delta^{-2 N a_{n}}\left(R^{n-N q}\right)^{1 / q} \\
& \lesssim \delta^{\left(a-2 n a_{n}\right) / q+(n-1) a_{n}}+\delta^{N \epsilon-\left(n \epsilon+2 n a_{n}\right) / q} \\
& \lesssim \delta^{\left(a-2 n a_{n}\right) / q+(n-1) a_{n}} .
\end{aligned}
$$

It follows that

$$
\left\|\widehat{\phi_{\delta} d \sigma}\right\|_{L^{q}\left(B^{c}\right)} \lesssim \delta^{a / p}
$$

whenever

$$
\frac{a}{p}+\frac{2 n a_{n}-a}{q} \leq(n-1) a_{n}
$$

which is larger than the range of exponents in (6). So we only need to worry about $\left\|\widehat{\phi_{\delta} d \sigma}\right\|_{L^{q}(B)}$.

We are going to use three basic estimates. The first is the Tomas-Stein estimate (see [2] and [3])

$$
\|\widehat{f d \sigma}\|_{L^{(2 n+2) /(n-1)\left(\mathbb{R}^{n}\right)}} \lesssim\|f\|_{L^{2}\left(\mathbb{S}^{n-1}\right)}
$$

for $f \in L^{2}\left(\mathbb{S}^{n-1}\right)$. The second is the local estimate

$$
\|\widehat{f d \sigma}\|_{L^{q}(B)} \lesssim R^{\frac{1}{2}\left(\frac{n+1}{q}-\frac{n+1}{2}+1\right)}\|f\|_{L^{2}\left(\mathbb{S}^{n-1}\right)}
$$

for $f \in L^{2}\left(\mathbb{S}^{n-1}\right)$ and $2 \leq q \leq(2 n+2) /(n-1)$, which is obtained by interpolating between (11) and the trace estimate

$$
\|\widehat{f d \sigma}\|_{L^{2}(B)} \lesssim R^{1 / 2}\|f\|_{L^{2}\left(\mathbb{S}^{n-1}\right)}
$$

To obtain the third estimate, we use a standard uncertainty principle argument.

Let $\psi \in C_{0}^{\infty}\left(\mathbb{R}^{n}\right)$ be such that $|\widehat{\psi}(\xi)| \geq 1$ for $|\xi| \leq 1$, and set

$$
\psi_{R}(x)=R^{n} \psi(R x) \quad \text { and } \quad \Psi=R^{-1} \psi_{R} *\left(\phi_{\delta} d \sigma\right) .
$$


Then $\Psi$ is supported in a box of center $e_{1}$ and dimensions $C \delta^{2 a_{2}} \times C \delta^{a_{2}} \times \cdots \times C \delta^{a_{n}}$ with the $\delta^{2 a_{2}}$ side parallel to $e_{1},\|\Psi\|_{L^{\infty}\left(\mathbb{R}^{n}\right)} \lesssim 1$, and

$$
\left\|\widehat{\phi_{\delta} d \sigma}\right\|_{L^{q}(B)} \lesssim R\|\widehat{\Psi}\|_{L^{q}\left(\mathbb{R}^{n}\right)} .
$$

Cover $\mathbb{R}^{n}$ by a finitely overlapping sequence $\left\{T_{l}\right\}$ of boxes such that each $T_{l}$ has dimensions $C \delta^{-2 a_{2}} \times C \delta^{-a_{2}} \times \cdots \times C \delta^{-a_{n}}$ with the $\delta^{-2 a_{2}}$ side parallel to $e_{1}$, and

$$
\sum_{l=1}^{\infty}\|\widehat{\Psi}\|_{L^{\infty}\left(T_{l}\right)}^{2}\left|T_{l}\right| \lesssim\|\Psi\|_{L^{2}\left(\mathbb{R}^{n}\right)}^{2} .
$$

(This is a straightforward application of the inequality

$$
|f(x)| \lesssim \sum_{|\alpha| \leq n+1}\left\|D^{\alpha} f\right\|_{L^{1}(B(x, 1))}
$$

and Plancherel's theorem.) Then

$$
\sum_{l=1}^{\infty}\|\widehat{\Psi}\|_{L^{\infty}\left(T_{l}\right)}^{2} \lesssim\left(\delta^{a+2 a_{2}}\right)^{2}
$$

Letting $c_{l}=\delta^{-2\left(a+2 a_{2}\right)}\|\widehat{\Psi}\|_{L^{\infty}\left(T_{l}\right)}^{2}$, we get

$$
|\widehat{\Psi}(\xi)|^{2} \lesssim \delta^{2\left(a+2 a_{2}\right)} \sum_{l=1}^{\infty} c_{l} \chi_{T_{l}}(\xi)
$$

with $\sum_{l=1}^{\infty} c_{l} \lesssim 1$. Thus

$$
\left\|\widehat{\phi_{\delta} d \sigma}\right\|_{L^{q}(B)} \lesssim R \delta^{a+2 a_{2}-\left(a+2 a_{2}\right) / q}=\delta^{a+2 a_{2}-2 a_{n}-\epsilon-\left(a+2 a_{2}\right) / q}
$$

provided $q \geq 2$.

Interpolating between (11) and the trivial estimate $\|\widehat{f d \sigma}\|_{L^{\infty}\left(\mathbb{R}^{n}\right)} \leq\|f\|_{L^{1}\left(\mathbb{S}^{n-1}\right)}$, we get (3) for $q \geq(2 n+2) /(n-1)$ and

$$
\frac{n-1}{p}+\frac{n+1}{q} \leq n-1
$$

Applying with $f=\phi_{\delta}$, we obtain

$$
\left\|\widehat{\phi_{\delta} d \sigma}\right\|_{L^{q}(B)} \lesssim \delta^{-\left(a_{n}+\frac{\epsilon}{2}\right)\left(\frac{n+1}{q}-\frac{n+1}{2}+1\right)} \delta^{a / 2}
$$

for $2 \leq q \leq(2 n+2) /(n-1)$. So to get

$$
\left\|\widehat{\phi_{\delta} d \sigma}\right\|_{L^{q}(B)} \lesssim \delta^{a / p}
$$

we need

$$
\frac{a}{p}+\frac{(n+1)\left(a_{n}+\epsilon / 2\right)}{q} \leq \frac{a+(n-1)\left(a_{n}+\epsilon / 2\right)}{2} .
$$

Now if $(p, q)$ is such that

$$
\frac{a}{p}+\frac{(n+1) a_{n}}{q}<\frac{a+(n-1) a_{n}}{2}
$$

we choose $\epsilon$ small enough for $(p, q)$ to satisfy (15), and (14) follows. Therefore, (3) holds whenever $(p, q)$ satisfies (6) $)$.

Finally, we notice that (13) tells us that (3) holds whenever

$$
\frac{a}{p}+\frac{a+2 a_{2}}{q}<a+2 a_{2}-2 a_{n}
$$


(compare with (4)). When $a_{2}=\cdots=a_{n}$, this becomes

$$
\frac{n-1}{p}+\frac{n+1}{q}<n-1 \text {. }
$$

This shows that one can get (7) without having to appeal to Tomas and Stein.

\section{REFERENCES}

1. W. Beckner, A. Carbery, S. Semmes and F. Soria, A note on restriction of the Fourier transform to spheres, Bull. London Math. Soc. 21 (1989), 394-398. MR 90i:42023

2. E. M. Stein, Harmonic Analysis: Real-Variable Methods, Orthogonality, and Oscillatory Integrals, Princeton University Press, Princeton, NJ, 1993. MR 95c:42002

3. P. Tomas, A restriction theorem for the Fourier transform, Bull. Amer. Math. Soc. 81 (1975), 477-478. MR 50:10681

Department of Mathematics, American University of Beirut, Beirut, Lebanon

E-mail address: bshayya@aub.edu.lb 